Title of Paper: $\quad$ Lost in Translation: Maximising handover effectiveness between paramedics and receiving staff in the emergency department

Authors:

Dr Christine Owen 60\%

Dr Lynn Hemmings 30\%

Dr Terry Brown 10\%

Institutions: $\quad$ Dr Owen - Faculty of Education, University of Tasmania

Dr Hemmings - Faculty of Education, University of Tasmania

Dr Brown - Postgraduate Institute of Tasmania

Author and address for correspondence :

Dr Christine Owen

Faculty of Education, University of Tasmania

Private Bag 66

Hobart TAS 7001

Ph: +61 362262555

Fax: +61362262569

Christine.Owen@utas.edu.au 


\title{
Lost in Translation: Maximising handover effectiveness between paramedics and receiving staff in the emergency department
}

\begin{abstract}
Objective: The purpose of this study is to investigate perceptions by paramedics and hospital receiving staff about what enables and constrains handover in the emergency department.

Methods: This is a qualitative study of interviews with 19 paramedics, 15 nurses and 16 doctors $(\mathrm{n}=50)$ from ambulance services and emergency departments in 2 states of Australia.

Results: Three main themes emerged that were evident at both sites and in the 3 professional groups. These were: difficulties in creating a shared cognitive picture, tensions between 'doing' and 'listening', and fragmenting communication. Conclusion: Recommendations arising from this study as to how handover could be improved are the need for a common language between paramedics and staff in the ED; for shared experiences and understanding between the members of the team, and, for the development of a standardised approach to handover from paramedics to ED receiving staff.
\end{abstract}

Keywords: handover, paramedic to emergency department interaction, patient safety, qualitative research 
In a recent issue in this journal, Yong, Dent and Weiland ${ }^{1}$ acknowledged that although emergency physicians are generally satisfied with paramedic handover, there is scope for improved doctor-paramedic communication at handover. The study by Yong et al is timely and highlights the paucity of research, particularly in Australia, on prehospital to emergency department (ED) handovers including a significant knowledge gap on staff perception of handovers and handover information. This is particularly important given the increasing recognition of the importance of effective clinical handover as a major determinant for patient safety ${ }^{2}$.

The purpose of this paper is to report on a qualitative study investigating perceptions by paramedics and receiving hospital nursing and medical staff about what enables and constrains effective handover in the ED and to develop recommendations to improve handover between paramedics and hospital receiving staff. The paper complements and extends the work of Yong et $\mathrm{al}^{1}$, in part because it provides insights into the limited knowledge currently available on the experiences of paramedics and receiving hospital medical and nursing staff about effective handover in the ED.

\section{Background}

Most of the literature on handover in the ED has focussed on nurse-to-nurse handover ${ }^{3}$ with fewer studies on handover between doctors ${ }^{4}$ and a scarcity of research on the transfer of information from ambulance to ED staff ${ }^{1}$. Problems which have been identified, however, include: the number of hands through which patients pass, the stressful and chaotic environment in the ED, the short period available for handover, lack of formal training in giving handover and difficulties in receiving staff 
retaining verbal information ${ }^{1,5,6,7,8,9,10}$. Recommendations to facilitate the orderly transfer and retention of information in the ED have included the development of a handover 'gold standard', the use of structured guidelines, minimization of the number of handovers on each patient and electronic data templates ${ }^{1,4,6,11,12}$.

\section{Methods}

Given the limited research conducted in this area a qualitative approach was chosen as the most appropriate methodology based on the assumption that this would have the potential to portray a full and rich account of the experiences from the hospital ED receiving staff and paramedics' frame of reference.

In keeping with the qualitative approach, selection of participants was opportunistic, and based on their first hand experience with the phenomenon of interest and willingness to participate in the study ${ }^{16}$. The participants were recruited from 2 hospitals and 2 ambulance services across 2 states. The services selected were of similar size and supported a metropolitan/regional population. The hospitals were both acute public teaching hospitals with 400 to 500 beds and busy emergency departments. Both ambulance services were within the catchment area of the 2 selected hospitals.

A semi-structured interview script was developed based on issues around handover identified in the literature. 3 experienced qualitative researchers conducted the interviews, using open-ended probing questions to elicit participants' perceptions of handover. Interviews were transcribed verbatim immediately following each interview with identifying data removed. The data analysis was guided by 
interpretational qualitative analysis which results in an organising system of data which are further refined to concepts or themes ${ }^{17}$. To enhance the reliability of analysis the 3 researchers independently assessed the transcripts before reaching a shared agreement. Early themes were revised and refined through a process of constant comparison of instances from the data and confirmed the direction of future interviews. Interviewing continued until no new information was found and the researchers believed that data saturation was achieved.

In total, 50 interviews were conducted with 19 'paramedics', 15 nurses and 16 doctors. The paramedics interviewed included ambulance officers, paramedics and intensive care paramedics with experience ranging from 2 to 15 years. As the level of training and position titles for these officers varied between the sites, it was decided that for the purposes of this study the term 'paramedics' would be used as a broad inclusive term to encompass this group. All of the nurses interviewed were Registered Nurses working permanently in ED with experience ranging from 3 years to 25 years. Of the doctors who participated in the study, 8 were ED consultants with a range of 6 years to 18 years, 6 were ED registrars and 2 were junior doctors rotating through the ED.

Prior to interviews, the study received Institutional ethics approval and informed consent was obtained from all participants.

To be relevant research concepts from a qualitative point of view, reliability and validity are conceptualised as rigor, with the goal of accurately representing what those who have been studied experience ${ }^{18}$. Within the context of this study, the 
researchers attempted to adhere as closely as possible to the techniques outlined by Guba $^{19}$ which support the rigor of the work - credibility, dependability, confirmability and transferability.

\section{Results}

The 3 core themes used in this paper were selected because they were pervasive across all 3 participant groups and both sites. These were:

1. Difficulties in creating a shared cognitive picture

2. Tensions between 'doing' and 'listening'

3. Fragmenting communication 'Chinese whispers'

\section{Difficulties in creating a shared cognitive picture}

Participants spoke of the difficulty they had in conveying and receiving information in a way that resulted in a shared cognitive picture. Paramedics in particular expressed frustration at how to report their perception of the patient in the pre-hospital context in such a way that it would be understood by receiving staff in the ED. As one paramedic explained:

I have to describe to a doctor or a nurse that the patient has been in a motor vehicle accident, high speed on this freeway, and they're lying back there smiling with a cervical collar on, looking okay... I have a lot of difficulty in being able to translate to them what the body has been through in the last hour because they're not seeing it...So that is the difficulty of using language to paint a picture for people when they weren't at the scene (Paramedic 4, Site 1). 
Receiving staff also spoke of the difficulty they had in trying to translate the information they hear from paramedics during handover. However, they stressed that the verbal report was just one of the ways they gathered information: You have a certain sense of urgency in that you're looking at what is giving you the most relevant information right there and then. Sometimes if you're not getting good information verbally from the paramedics you're looking for written information or you're looking for visual information... anything to help get an understanding of the situation (Doctor 3, Site 1).

One of the issues that both paramedics and receiving staff said contributed to the difficulty in reaching a shared view of the patient during handover was the lack of a shared language. As one paramedic observed:

...what you've got is the complexity of how you translate your picture of that scene to another person who may not be speaking your language (Paramedic 7, Site 1).

This view was shared by many of the participants who agreed that using a ‘common language' was an essential component of a good handover: We're not really good with the lingo...the language that we use is often different. They (paramedics) tend to use militaristic language even in the way they describe symptoms of patients... it all adds to unnecessary confusion during handover (Nurse 10, Site 2).

\section{Tensions between doing and listening}

A frequent source of tension for paramedics was their experience of the receiving staff physically attending to the patient during the handover rather than 
listening. They gave many examples of their frustration of just getting someone to listen even for a few minutes while they presented the patient:

Nurses are multi tasked so they are trying to do 4 or 5 things at once so they are not being overtly attentive, or not listening at all, or are continuously interrupting your train of thought by getting you to move the patient across or do this or do that and then getting you to restart the handover again. (Paramedic 10, Site 1).

In some cases, paramedics spoke of keeping the patient on 'their stretcher' while giving handover as this often increases the chance that the receiving staff will 'stop and listen'. One paramedic explained that once he had transferred a patient on the ED trolley he felt he had 'lost the upper hand':

It's an issue everywhere with ambulance in that we've had the patient for half an hour and we know what's happening with them...they (receiving staff) have an immediacy in that the patient they have been waiting for is here and they want to start doing things. So it's a point of friction in that we would prefer that patients stayed on our stretcher until we've handed them over so that basically they have to listen to us, and then they can have the patient - they want the patient on their trolley so they can start doing stuff...(Paramedic 2, Site 1).

There was acknowledgement by receiving staff that they did not always listen attentively during handover. They highlighted, however, that they had multiple tasks they had to attend to whereas the paramedic only had one task during handover. Several medical staff spoke of the difficulty in concentrating during handover with so many distractions and competing demands. For instance one junior doctor spoke of 
needing to find a balance between getting involved with the patient and listening to the paramedic:

One of the things I find difficult is that when you're getting handover there's a lot of other stuff happening at the same time, and it's distracting. It's important to concentrate on listening to the handover, but at the same time, there are other things that need to be done simultaneously, and I guess it's a balance between how much do you focus on just getting the handover and leave everything to everyone else, or how much do you keep an eye on what's going on while listening to the handover? (Doctor 6, Site 1)

Despite an awareness by receiving staff that they often did not listen attentively during handover, there was agreement that handover formed an important part of the overall decision making process. Medical staff in particular expressed concern that if receiving staff did not listen that the details, nuances and vital clues that were contained within handover could be lost. They suggested that despite the sometimes chaotic atmosphere during handover that paramedics had a responsibility to ensure their message is heard by being assertive, speaking loudly and ensuring that there was a clear leader in the process. For instance, an experienced ED doctor suggested:

There's a lot of voices all at once but if the paramedics speak loudly and hold the floor just for that brief moment or two to make it clear what has happened and what the patient has been like I think it sets the scene and establishes order (Doctor 14, Site 2).

\section{Fragmenting communication - 'Chinese Whispers'}


The colloquial term 'Chinese whispers' was used, unprompted in over 20 interviews to describe how information changed during the handover process. As one paramedic commented 'there's a lot lost in translation'. The concept of information being 'lost in translation' was also noted by Solet, Norvell, Rutan and Frankel in a study of physician to physician handover ${ }^{20}$.

Most participants felt that the lack of a structured process for presenting information contributed to the problem:

We end up with Chinese whispers... you've got a paramedic operating at the peak of their clinical performance handing over to an officer who may not have worked operationally for a number of years...Then they're trying to translate that information to the hospital, and in the absence of a structured process that information will change along the way... the nuances and urgencies aren't being picked up by the various groups...so we end up with Chinese whispers (Paramedic 6, Site 1).

A junior doctor expressed his frustration about a lack of structure to the handovers he received:

Some of the paramedics are great but some just go on and on and it's all waffly. It would be good to have a consistent way that handovers are given so they are to the point, but cover all the necessary information. And then we also need some teaching on how to accept the handovers. It's just assumed that they (paramedics) can give it and we can listen...but it doesn't always happen like that (Doctor 8, Site 2). 
There was agreement by the participants that the multiple times that handover could be repeated contributed to the problem of information being lost or changed during the process.

It's almost like there's a handover on the mobile, a handover when you get to triage, handover to the nursing staff who's actually going to be looking after them or to another ambo who's going to be looking after them in the meantime, then the nurse to the doctor. It just goes on and on and on...It's a funny area, what this whole handover thing and it's hard to keep a consistent story (Paramedic 7, Site 1).

\section{Discussion}

Our data support the findings of Yong et $\mathrm{al}^{1}$ but also extends this work by identifying the nature of difficulties in interdisciplinary communication in this environment. The findings illustrate that the emergency department is a complex environment where there are difficulties in both handing over and receiving information. These difficulties occur due to differences in language, lack of clear leadership, distractions while listening to the handover and the distortion of verbal information as it is transmitted to other health care members. Our findings suggest the following recommendations:

\section{Need to develop a common language}

The fact that the teams involved in handovers in the emergency department are comprised of members from a variety of different disciplines can result in variations in communication styles and language. Studies show that not only improvements in communication processes lead to increases in patient safety, but also that when communication problems do occur, they are found most often between different 
members of a team ${ }^{21,22}$. Differences in language, of even a minor nature, can cause difficulty in understanding 'the finer points of meanings, intentions and reactions, ${ }^{2,15}$. It is therefore essential that all members of the handover team share a common language for effective team communication.

\section{$\underline{\text { Need to develop shared experiences }}$}

The interdependent nature of handover in the ED requires that there is a high level of shared understanding among the members of the team about their respective roles, tasks and objectives throughout the handover process ${ }^{22}$. Paramedics and ED staff largely operate in different environments, coming together only momentarily. This results in a lack of awareness of each other's duties, responsibilities and problems. Organisational factors such as administrative segregation and differences in training also contribute to the differences across the disciplines ${ }^{23}$.

Research suggests ${ }^{3,4,6,22,24}$ that the more that team members know about each others roles, the more awareness they will have of each other's tasks and objectives, and in turn, the more efficient the team will become. One way that a shared understanding can be achieved is through shared experiences such as interdisciplinary training, utilising simulated scenarios to provide opportunities for trainees to demonstrate teamwork and an understanding of each others roles. This results in an effective form of education for ED staff, including training in non-technical skills such as listening within a complex environment ${ }^{24}$. Although there was a lack of interdisciplinary training amongst the participants in this study, they were enthusiastic about the potential benefits. 


\section{Need for standardised approach}

Paramedics in this study indicated that a lack of formal training in giving a standardised handover resulted in difficulties in achieving a comprehensive presentation of information. Receiving ED staff also noted that this was a barrier to effective handover. While the recent AMA guidelines on clinical handover acknowledges that 'the style of handover will vary depending on local need', it recommends that 'all types need a predetermined format and structure to ensure adequate information exchange, ${ }^{2}$. A predetermined format also reduces the likelihood of information being distorted as it moves between health professionals. The results reported here support the need for both paramedics and ED receiving staff to have training in the minimum amount of information required in a handover. Ye et al suggests that a handover 'gold standard' would help to standardise information handed over in the $\mathrm{ED}^{4}$. It is important to note, however, that while participants in this study were generally supportive of some form of standardisation of handover, they wanted to retain a degree of flexibility and recognition of the professional expertise and judgement of those involved.

\section{Limitations to the study}

There are several limitations to this study related to the general limitations of qualitative research. Judged against quantitative criteria, the sample size is relatively small and the results must therefore be interpreted with caution. However, even though the findings cannot be generalised across all emergency departments, they have provided insights that can be used for larger, quantitative studies. Another limitation to this study is that health professionals were asked questions concerning their peers who they work with on a daily basis, which may have influenced their 
responses. To minimize this influence, the researchers reassured the participants that their responses would be confidential and any reported data would be anonymised.

An important goal of future research is to determine if the factors contributing to effective handover outlined in the literature, including the recommendations in this paper, actually result in improved handover. It is clear that ongoing qualitative and quantitative research is necessary to determine what interventions are most effective in improving safety and quality of handover between paramedics and ED receiving staff. (Is this wording OK and should it be here or in another section??)

\section{Conclusion}

'The aim of any handover is to achieve the efficient communication of high quality clinical information at any time when the responsibility for patient care is transferred' ${ }^{1}$. Our findings indicate that while paramedics and receiving staff in the ED recognise the importance of effective handover, there are a number of factors that result in a variable quality of handover. Barriers to effective handover need to be acknowledged and addressed to ensure continuity of information vital to the safety of patients in the emergency department. 


\section{References}

1. Yong G, Dent AW, Weiland T. Handover from paramedics: observations and emergency department clinician perceptions. Emerg. Med. Australas. 2008; 20: $149-155$.

2. Australian Medical Association. Safe handover: safe patients - guidance on clinical handover for clinicians and managers. Canberra: AMA; 2006.

3. Currie J. Improving the efficiency of patient handover. Emerg. Nurse. 2002; 10: $24-27$.

4. Ye K, Taylor D, Knott J, Dent A, MacBean E. Handover in the emergency department: deficiencies and adverse events. Emerg. Med. Australas. 2007; 19(5): 433-441.

5. Victorian Department of Human Services. Report of the Ministerial Taskforce on Trauma and Emergency Services and the Department of Human Services Working Party on Emergency and Trauma Services, Trauma and Emergency Services. Melbourne: Victorian Department of Human Services; 1999.

6. Jenkin A, Abelson-Mitchell N, Cooper S. Patient handover: time for a change? Acci. Emerg. Nurs. 2007; 15(3): 141-147. 
7. Talbot R, Bleetman A. Retention of information by emergency department staff at ambulance handover: Do standardised approaches work? EMJ. 2007; 24: 539-542.

8. Scott L, Brice J, Baker C, Shen P. An analysis of paramedic verbal reports to physicians in the emergency department trauma room. Prehosp. Emerg. Care. 2003; 7(2): 247-251.

9. Thurgood G. Verbal handover reports: what skills are needed? Br. J. Nurs. 1995; 4(12): 720-722.

10. Thakore S, Morrison W. A survey of the perceived quality of patient handover by ambulance staff in the resuscitation room. EMJ. 2001;18: 293-296.

11. Australian Council for Safety and Quality in Health Care. Passing the baton of care - the patient relay. Sydney: Office of Australian Council for Safety and Quality in Health Care. 2005.

12. Bruce K, Suserud B. The handover process and triage of ambulance-borne patients: The experiences of emergency nurses. Nurs. Crit. Care. 2005; 10(4): 201-209.

13. Cooper S, Endacott R. Generic qualitative research: a design for qualitative research in emergency care? EMJ. 2007; 24: 816-819. 
14. Britten N. Qualitative research: qualitative interviews in medical research. BMJ. 1995; 311: 251-253.

15. Dixon-Wood M, Fitzpatrick R. Qualitative research in systemic reviews has established a place for itself. BMJ. 2001; 323: 765-766.

16. Patton M. Qualitative evaluation and research methods. Beverly Hills: Sage; 1990.

17. Tesch R. Qualitative research: analysis types and software tools. New York: The Falmer Press; 1990.

18. Golafshani N. Understanding Reliability and Validity in Qualitative Research. TQR. 2003; 8(4): 597-607

19. Guba EG. Criteria for assessing the trustworthiness of naturalistic inquiries. Educational Communication and Technology Journal. 1981; 29: 75-92.

20. Solet, D.J., Norvell, J.M., Rutan, G.H., \& Frankel, R.M. Lost in translation: challenges and opportunities in physician to physician communication during patient handoffs. Acad Medicine. 2005; 80(12): 1094-9.

21. Davies JM. Team communication in the operating room. Acta Anaesthesiol Scand. 2005; 49: 898-901. 
22. Undre S, Sevdalis N, Healey A, Darzi A, Vincent C. Teamwork in the operating theatre: cohesion or confusion? J Eval Clin Pract. 2006; 12(2): 182189.

23. Hammon $\mathrm{W}$. The complexity of team training: what we have learned from aviation and its applications to medicine. Qual. Saf. Health Care. 2004; 13(1): 172-179.

24. Shapiro M, Morey J, Small S, et al. Simulation based teamwork training for emergency department staff: does it improve clinical team performance when added to an existing didactic teamwork curriculum? Qual. Saf. Health Care. 2004; 13: 417-421. 